\title{
Attention Training Toward and Away from Threat in Social Phobia: Effects on Subjective, Behavioral, and Physiological Measures of Anxiety
}

\section{Citation}

Heeren, Alexandre, Hannah Elizabeth Reese, Richard J. McNally, and Pierre Philippot. 2012. Attention training toward and away from threat in social phobia: Effects on subjective, behavioral, and physiological measures of anxiety. Behaviour Research and Therapy 50(1): 3039.

\section{Published Version}

doi:10.1016/j.brat.2011.10.005

\section{Permanent link}

http://nrs.harvard.edu/urn-3:HUL.InstRepos:8916507

\section{Terms of Use}

This article was downloaded from Harvard University's DASH repository, and is made available under the terms and conditions applicable to Open Access Policy Articles, as set forth at http:// nrs.harvard.edu/urn-3:HUL.InstRepos:dash.current.terms-of-use\#OAP

\section{Share Your Story}

The Harvard community has made this article openly available.

Please share how this access benefits you. Submit a story.

Accessibility 


\title{
Running head: ATTENTION TRAINING IN SOCIAL PHOBIA
}

A randomized controlled trial of attention training in social phobia:

Effects on behavioral, subjective, and physiological measures

\author{
Alexandre Heeren ${ }^{\text {a, b }}$, Hannah E. Reese ${ }^{\mathrm{c}}$, Richard J. McNally ${ }^{\mathrm{d}}, \&$ Pierre Philippot ${ }^{\mathrm{a}}$ \\ ${ }^{a}$ Université catholique de Louvain, Louvain-la-Neuve, Belgium \\ ${ }^{\mathrm{b}}$ National Fund for Scientific Research, Brussels, Belgium \\ ${ }^{\mathrm{c}}$ Department of Psychiatry, Massachusetts General Hospital, Boston, USA \\ ${ }^{\mathrm{d}}$ Harvard University, Cambridge, USA \\ Behaviour Research and Therapy (in press)
}

Author note

This work was supported by a grant from the Belgian National Fund for Scientific Research, awarded to Alexandre Heeren (1.1.315.09.F), and by a Joined Research Grant (ARC 06/11-337) from the Belgian French Community, awarded to Pierre Philippot.

Correspondence concerning this article should be addressed to Alexandre Heeren or Pierre Philippot at the Institute of Psychological Science, Université catholique de Louvain, Place du Cardinal Mercier, 10, B-1348 Louvain-la-Neuve, Belgium. Electronic 
mail may send via Internet to "Alexandre.Heeren@uclouvain.be“"or

“Pierre.Philippot@uclouvain.be

\begin{abstract}
Social Phobics exhibit an attentional bias for threat in probe detection and probe discrimination paradigms. Attention training programs, whereby probes always replace nonthreat cues, reduce attentional bias for threat and self-reported anxiety. However, it remains unclear whether the therapeutic benefits of attention training result from people learning to disengage attention from threat cues or acquiring greater control over their attention by learning to deploy it flexibly. Moreover, researchers have seldom taken behavioral measures, and have never taken physiological measures of fear reduction. Investigating these questions, we found that training to disengage attention from threat is more effective than training to deploy it flexibly in social phobia. Indeed, the former condition reduced self-report, behavioral and physiological measures of anxiety.
\end{abstract}


A randomized controlled trial of attention training in Social Phobia:

Effects on behavioral, subjective, and physiological measures

Most cognitive models of anxiety propose that selective attention to threat cues contributes to the development and maintenance of emotional disorders (e.g., Mathews \& MacLeod, 1994). For people with social phobia, these cues include threatening facial expressions displaying anger or disgust and words signifying social threat (e.g., humiliation). In probe detection and probe discrimination tasks, subjects with social anxiety or social phobia are faster to respond to probes that replace these cues than those that replace neutral cues, thereby exhibiting an attentional bias for threat that is absent in nonanxious control subjects (e.g., Mogg, Philippot, \& Bradley, 2004; Pishyar, Harris, \& Menzies, 2004). It remains unclear whether attentional biases for threat arise because threat cues rapidly capture attention, delayed disengagement, or both (Yiend, 2010).

More recently, studies involving a modified Posner (1980) spatial cuing paradigm showed that anxious participants are no faster to respond to probes replacing threat than nonthreat cues, but they are slower to respond to probes that appear opposite to threat cues relative to nonthreat ones, implying that anxious participants have difficulty disengaging attention from threat (e.g., Amir, Elias, Klumpp, \& Przeworski, 2003). In this paradigm, a threat (or nonthreat) cue appears on either the left or right side of a computer screen, and a probe either replaces the cue or appears on the other side of the screen (e.g., Amir et al., 2003; Fox, Russo, Bowles, \& Dutton, 2001).

Attentional bias for threat has consequences. Its reemergence predicts the return of anxiety at follow-up among patients treated for generalized anxiety (Mogg, Bradley, Millar, \& White, 1995) and social phobia (Lundh \& Öst, 2001). Moreover, it causally 
influences anxiety proneness (MacLeod, Rutherford, Campbell, Ebsworthy, \& Holker (2002). Using a dot-probe detection task, MacLeod and colleagues trained participants to attend either to neutral or to threatening stimuli. The task comprised 672 trials in which pairs of words (one threatening and one neutral) appeared on a computer screen. In the attend threat condition, probes replaced threat words, whereas in the attend neutral condition, probes replaced neutral words. Participants pushed a button as soon as they detected the probe. Relative to those trained to attend to neutral material, participants trained to attend to threat material reported a greater increase in anxiety and in negative mood after performing a stressful anagram task. This study provides causal evidence that selective attention to negative information increases anxiety reactivity to an experimental stressor.

Regarding social phobia, Li, Tan, Qian, and Liu (2008) observed that, in comparison to a control condition, 7-days of attention training toward positive faces diminished attentional bias for negative faces and reduced self-reported fear of social interaction. Similarly, Amir, Weber, Beard, Bomyea, and Taylor (2008) trained, in a single-session, socially phobic individuals to attend either to non-threatening faces or to disgust faces. As compared to the latter condition, the former reduced anxiety in response to an impromptu speech. Blind raters judged the speeches of those in the no-threat attention training group more positively than those of the control group. Further, using a modified Posner (1980) paradigm after attention training, these authors observed that the improvement in the ability to disengage attention from threat mediated the effects of the training on anxiety reactivity, and that this decrease in anxiety, in turn, improved speech performance. Likewise, Schmidt, Richey, Buckner, and Timpano (2009) have observed 
that training individuals with social phobia to attend to neutral faces led to a significant reduction in social anxiety and trait anxiety, in comparison to a control group trained to attend disgust faces. At a 4-month follow-up, the treatment group had improved further on measures of anxiety. Recently, Amir, Beard, Taylor, Klump, Elias, Burns, and Chen (2009) have replicated these results.

These studies suggest that reducing attentional bias for threat can diminish emotional vulnerability to subsequent stressors. However, several issues require further examination. First, in most studies, researchers have assessed the impact of attention training by relying on questionnaire measures of anxiety and diagnostic interviews. Some researchers have assessed treatment response by including behavioral measures of fear (e.g., Amir et al., 2008; Klumpp \& Amir, 2010; Reese, McNally, Najmi, \& Amir, 2010), but none has incorporated physiological measures of fear. As MacLeod, Koster, and Fox (2009) argued, the completion of self-report measures involves judgment and inferences, giving rise to the possibility that a cognitive manipulation might affect questionnaire scores even when emotional experience itself is unaffected. Accordingly, as these authors suggested, researchers should supplement self-report measures with behavioral and physiological measures of anxiety. Hence, in the present study, we included self-report, behavioral, and physiological measures of anxiety to assess the effects of attention training in people with social anxiety disorder.

Uncertainty abounds regarding the mechanisms that mediate the reduction of emotional vulnerability via attention training. According to Amir et al. (2008), the improvement of the ability to disengage attention from threatening stimuli mediates the reduction of emotional reactivity to stressors (disengagement hypothesis). As mentioned 
above, studies show that anxious participants are no faster to respond to probes replacing threat than nonthreat cues, but they are slower to respond to probes that appear opposite to threat cues relative to nonthreat ones, implying that anxious participants have difficulty disengaging attention from threat (e.g., Amir et al., 2003; Fox et al., 2001). Hence, by extension, the mechanism mediating attentional bias for threat in the probe detection and probe discrimination paradigms may be disengagement difficulty (but see Mogg, Holmes, Garner, \& Bradley, 2008 for another account). In contrast, Wells (2000) holds that anxious people have difficulties deploying attention flexibly. Moreover, he suggested that this difficulty, regardless of the availability of resources, could contribute to cognitive vulnerability and maintenance of emotional disorders (flexibility hypothesis). According to the flexibility hypothesis, the effects of attention training may be boosted by training social phobics to flexibly attend to both threatening and non-threatening stimuli. The core process would then be to de-rigidify the automatic capture and maintenance of attention by threatening cues.

In the present double-blind experiment, we addressed two primary issues. First, we investigated the effects of attention training in social phobics on self-report, behavioral, and physiological measures of stress during a speech performance. Second, we examined whether training social phobics to attend flexibly to either threatening or nonthreatening stimuli would be more effective than standard attention training in reducing stress responses during the speech task. That is, we asked whether training attentional flexibility is more effective than training to disengage attention from threatening stimuli. 
We randomly assigned participants diagnosed as having social phobia to one of three attention training conditions: 1) attend to threat stimuli, 2) attend to neutral stimuli, or 3) attend alternatively to threat and neutral stimuli. Our primary prediction was that, compared with participants who were trained to attend to threatening stimuli, those trained to attend to non-threatening stimuli, and those trained to flexibly attend to both threatening and non-threatening stimuli would present (a) a decrease in attentional bias toward threat, (b) lower self-reported social anxiety, (c) lower physiological and subjective responses to a social stressor, and (d) improved behavioral performance during the speech task. According to the flexibility hypothesis, these effects would be stronger for participants trained to attend to both threatening and non-threatening stimuli. In contrast, the disengagement hypothesis predicts more beneficial outcomes for those trained to attend to non-threatening stimuli.

\section{Method}

\section{Overview and General Procedure}

Participants came to the laboratory for six visits. At the baseline visit, participants completed two self-report measures of social anxiety, a dot-probe assessment of attentional bias, and a stressful speech task. We then randomly assigned participants to receive one of three attentional training conditions: Attention to non-threatening stimuli, attention to threatening stimuli, or attentional flexibility. Neither the participant nor the experimenter was aware of the assigned training condition. Each training was delivered in 4 sessions over 4 consecutive days. Immediately after the final training session, participants repeated the assessment of self-reported social anxiety, dot-probe assessment of attentional bias, and the stressful speech task. Finally, participants 
returned to the laboratory two weeks after the final training session for assessment of self-reported social anxiety and debriefing.

\section{Participants}

We recruited 60 individuals with a primary DSM-IV (American Psychiatric Associations, 1994) diagnosis of Generalized Social Anxiety Disorder from the Université Catholique de Louvain community. A total of 213 volunteers responded to our invitation to take part in an investigation of the mechanisms underlying social interaction among shy people. Seventy-eight individuals met the initial eligibility criteria assessed via a screening questionnaire and subsequently completed a structured interview to assess diagnostic eligibility. To confirm the diagnosis of Social Anxiety Disorder, we administered the social phobia section of the Mini International Neuropsychiatric Interview (MINI; Lecrubier, Weiller, Bonora, Amorin, \& Lépine, 1994). Fifteen of the 78 pre-selected volunteers did not meet criteria for social anxiety disorder and 3 refused to participate. The remaining 60 participants were included in the study; their characteristics appear in Table 1. In addition to a primary diagnosis of Social Anxiety Disorder, all participants (a) had no current substance abuse, (b) no current or past heart, respiratory, neurological problems or use of psychotropic medications, (c) were not currently engaged in any form of psychological or psychiatric treatment and d) had normal or corrected-to-normal vision. Each participant was tested individually in a quiet room, and all attention training sessions were completed in the same laboratory. Participants received compensation (12.5 euros and a lottery ticket) for their participation. We conducted the study in accordance with the ethical standards of the American Psychological Association. 
Insert Table 1 about here.

\section{Measures}

Questionnaires. To characterize our participants, we asked them to complete the Trait Anxiety Inventory (STAI-Trait; Spielberger, Gorsuch, Lushene, Vagg, \& Jacobs, 1983) and the Beck Depression Inventory (BDI-II; Beck Steer, \& Brown, 1996) at the beginning of the first training session.

The STAI-Trait is a 20-item self-report questionnaire assessing trait proneness. Bruchon-Schweitzer and Paulhan (1993) have reported good psychometric and structural properties of the French adaptation of the scale. Cronbach's alpha in the current sample was .89 .

The BDI is a 21-item self-report measure of symptoms of depression. Beck, Steer, and Brown (1998) have reported good psychometric and structural properties of the French adaptation of the scale. Cronbach's alpha in the current sample was .82 .

Participants completed two self-reported scales of social anxiety at baseline, posttraining, and follow-up: the Liebowitz Social Anxiety Scale (LSAS; Liebowitz, 1987) and the Fear of Negative Evaluation scale (FNE; Watson \& Friend, 1969).

The LSAS is a 24-item scale that measures the anxiety induced by, and the avoidance of social interaction and performances situations. Yao, Note, Fanget, Albuisson, Bouvard, Jalenques, \& Cottraux (1999) have reported good psychometric and structural properties of the French adaptation of the scale. Cronbach's alpha in the current sample was .91.

The FNE is a 30-item self-report questionnaire that measures a person's apprehension about negative evaluation. Studies have reported good psychometric 
properties as well as structural validity of the French adaptation of the scale (Douilliez, Baeyens, \& Philippot, 2008; Musa, Kostogianni, \& Lépine, 2004). Cronbach's alpha in the current sample was .93 .

Measure of attention bias: Probe discrimination task. Participants completed a probe discrimination task modeled on the dot probe detection task (MacLeod, Mathews, \& Tata, 1986). The task measured attentional bias for threatening information at baseline and post-training. The task consisted of 96 trials delivered in one block. Each trial started with a central fixation cross which appeared on the screen for $500 \mathrm{~ms}$. Immediately following the disappearance of the cross, a pair of faces appeared on the screen for $500 \mathrm{~ms}$. Immediately following their disappearance, a probe (i.e., white arrow), pointing either up or down, replaced one of the faces. The probe remained on the screen until the participant indicated the direction of the arrow by pressing by a button. The inter-trial interval was $1500 \mathrm{~ms}$. An attentional bias for threat was demonstrated by a significantly faster response when the probe appeared in the location previously occupied by a threatening face than when the probe appeared in the location previously occupied by a neural face. There were an equal number of trials in each condition as a function of emotional face location (left or right) and probe type (up or down arrow). Each of 24 face pairs appeared four times representing all combinations of the locations and probe types (96 trials $=24$ faces pairs X 2 positions X 2 arrow's directions). The same pairs of faces appeared in a different random order for each participant. Participants completed eight practice trials (including four men and four women neutral face pairs) prior to the experimental trials. During the practice trials, participants received feedback regarding the accuracy of their response. No feedback occurred during the experimental trials. Participants sat approximately $30 \mathrm{~cm}$ from the computer screen. 
Stimuli consisted of 24 face pairs selected from the FaceGen Modeller (Singuar Inversions Inc, 2008) generated faces as described in the materials section (see below) and different from those used during the training procedure. The same face pairs were used at baseline and at post-training.

Speech task. We administered a speech task to assess self-report, physiological, and behavioral responses to a social stressor at baseline and post-training. Each participant began the task, sitting in a comfortable chair $30 \mathrm{~cm}$ from a computer screen. We then attached the skin conductance electrodes and instructed the participant to read the instructions that appeared on the screen. The first instruction read, "Calmly rest until another slide occurs" and appeared on the screen for 1 minute. Skin conductance was recorded during this $1 \mathrm{~min}$ baseline. The second set of instructions then appeared and informed participants that they would have to make a 2-minute speech about a negative emotional experience and that their performance would be video recorded. Two different topics (a negative experience with a friend or a negative academic experience) were randomly counterbalanced between times of assessment. This instruction remained on the screen for 2 minutes and skin conductance was recorded during this time. The final set of instructions then appeared on the screen, asking the participant to wait for the experimenter. The experimenter then directed participants to stand in a designated area, in another room, in front of a video camera. Just before starting the speech, the experimenter asked participants to rate their level of situational anxiety from 0 (not anxious) to 100 (extremely anxious) (Subjective Units of Discomfort Scale (SUDS); Wolpe, 1958). The participant then performed the speech while being videorecorded. 
Physiological response. Skin conductance reactivity (SCR) was measured with two $\mathrm{Ag}-\mathrm{AgCl} \mathrm{SCR}$ electrodes attached to the volar surfaces of the medial phalanges of the middle and ring fingers of the nondominant hand. Grass skin conductance paste (with the recommended 0.05-M NaCl saturation; Grey \& Smith, 1984) was the electrolyte. SCR We used a BIOPAC MP150 unit running Acqknowledge 4.0.0 software (Biopac Systems, 2008) with one SCR 100B amplifier to collect SCR data. The SCR amplifier had a sensitivity of $5 \mu \mathrm{ohm} / \mathrm{V}$, with a $10-\mathrm{Hz}$ low-pass filter and a $0.05-\mathrm{Hz}$ high-pass filter.

Behavioral assessment. Speech performance was rated by two judges, with at least three years of CBT training, who were blind to training condition. The rating scheme was the Behavioral Assessment of Speech Anxiety (BASA; Mulac \& Sherman, 1974), which includes 18 molecular categories (e.g., having a clear voice, searching for the words). The total score of these categories has shown excellent concurrent validity with experts' ratings of speech anxiety (Mulac \& Sherman, 1974). Inter-rater reliability of the total score was high $(r=.81$ at baseline), suggesting that a mean score of the two raters may be computed. Internal consistency of the data in our study was good $(\alpha=.73)$.

Attention training. Attention training consisted of the probe discrimination task described above, modified to promote either: 1) an attentional bias away from threatening material (AnT), 2) an attentional bias toward threatening material (AT), or 3) attentional flexibility (AF). In the AnT condition, the arrow always appeared in the location previously occupied by the non-threatening face. In the AT condition, the arrow always appeared in the location previously occupied by the threatening face. In the AF condition, an A-B-A-B design was used. Two blocks of differing trial types (no break between blocks) were alternated. In the first block, the probe replaced the threatening 
face in $80 \%$ of the trials and the non-threatening face in $20 \%$ of the trials. In the second block, the probe replaced the threatening face in $20 \%$ of the trials and the nonthreatening face in $80 \%$ of the trials. The switch occurred after every 200 trials.

Participants completed 744 trials per training session. Each of the 62 threatening faces was randomly paired with a non-threatening face, and was presented four times, representing all combinations of the locations and probe types, and this procedure was repeated 3 times (i.e., $744=62$ stimuli X 2 positions X 2 arrow's directions X 3 repetitions). All three groups were exposed to the same face pairs although the order of presentation was randomly determined for each subject.

\section{Materials}

Eighty-six pairs of faces were used in the dot-probe testing and in the attention training tasks. The face pairs were created with FaceGen 3.1 software (Singular Inversions Inc., 2008) that is based on statistical modeling of a sample of real faces varying in ethnicity, age, and gender. To model faces, the software uses more than 100 dimensions, such as eye, mouth, or lip size. We generated 20 angry faces for both genders and for three ethnic groups (Caucasian, African, and Asian), resulting in 120 faces. We pretested these threatening faces (on a scale from $1=$ absolutely not threatening to $9=$ absolutely threatening) on 19 college students. We selected the 86 faces expressing anger most clearly. Among the selected faces, there were no ethnic group or gender differences in anger ratings.

We then generated non-threatening stimuli by duplicating each angry face and manipulating the facial features to possess a $40 \%$-level of closed smile expression. The use of light smile faces as non-threatening stimuli was based on previous works 
suggesting that socially anxious individuals tend to interpret neutral faces as threatening (e.g., Sommerville et al., 2004; Yoon \& Zinbarg, 2008).

Each face pair consisted of the same individual displaying either an angry or a light smile expression. Each picture was $11 \mathrm{~cm}$ high and $7.6 \mathrm{~cm}$ wide. Faces were separated by $11.5 \mathrm{~cm}$ from their centers. All stimuli appeared against a black background.

\section{Results}

Six participants were excluded from the analyses, three (one from the AT condition and two from the AnT condition) missed one training session, one, from the AF condition, got sick during training, and two, one from the AT condition and one from the AF condition, dropped-out without explanation. All statistical analyses were conducted on the 54 remaining participants.

\section{Group Equivalence}

Preliminary analyses indicated no differences among the groups at baseline on STAItrait, $F(2,51)=2.54, p>.09$, BDI-II, $F(2,51)=2.49, p>.09$, LSAS, $F(2,51)=.08, p$ $>.93$, and FNE, $F(2,51)=.37, p>.69$. All groups were similar in terms of age $F(2,51)$ $=.27, p>.76$, gender, $\chi^{2}(2, \mathrm{~N}=54)=1.11, p>.57$, and years of education, $F(2,51)=$ $.67, p>.52$.

\section{Data Analyses}

Separate multivariate analysis of variance (MANOVAs), with Time (baseline, post-training) as a within-subject factor and Condition (AT, AnT, AF) as betweensubjects factor, were computed on the measure of attention bias and on the behavioural, electrodermal, and subjective measures of emotional reactivity during the speech task. For self-reported measures of social anxiety, we computed separate MANOVAs, with 
Time (baseline, post-training, follow-up) as a within-subject factor and Condition (AT, AnT, AF) as between-subjects factor, and we applied Greenhouse-Geisser corrections when necessary (Mauchley's Sphericity Test $<.05$ ). We used paired-sample $t$ tests to probe interactions.

\section{Independent measure of attentional bias}

Data reduction. Latencies from trials with errors were excluded (2 \% of the data). Data more than two standard deviations below or above the participant's mean were discarded as outliers ( $1 \%$ of the data). At baseline, the three groups did not differ significantly in error rates, $F(2,51)=.49, p>.61$. As the primary variable of interest was vigilance for or avoidance from emotional faces, we calculated a $d$ (or bias) score for each participant (MacLeod \& Mathews, 1988) by subtracting the mean latency when the probe appeared at the same location as the threatening face from the mean latency when the probe and threatening face appeared at different locations (see Table 2). A positive bias score indicates faster detection of probes replacing threatening faces (i.e., vigilance for threat). To test whether there were any group differences before training, we conducted a one-way ANOVA on the $d$ values. Results indicates no significant differences among the groups at pre-training, $F(2,51)=.107, p>.90$.

\section{Insert about here Table 2}

\section{Change in attentional bias. The MANOVA revealed a significant Time X}

Condition interaction, $F(2,51)=7.56, p<.01, \eta_{\mathrm{p}}{ }^{2}=.29$. In the AT condition, there were no significant change from baseline to the post-training, $t(17)=.17, p>.87$. In the AnT condition, there was a significant decrease from baseline to post-training, $t(17)=7.74, p$ 
$<.01$. In the AF condition, there was no significant change from baseline to the posttraining, $t(17)=1.116, p>.28$.

Self-reported measures of Social Anxiety

For the LSAS, the MANOVA revealed a main effect of Time, $F(2,51)=17.01, p$ $<.001, \eta_{\mathrm{p}}{ }^{2}=.25$, qualified by a significant Time X Condition interaction, $F(1,51)=$ $5.20, p<.01, \eta_{\mathrm{p}}{ }^{2}=.17$. AT participants showed no differences from baseline to both post-training, $t(17)=.74, p>.47$, and follow-up, $t(17)=1.83, p>.08$. In contrast, AnT participants reported significant decreases in social anxiety from baseline to both posttraining, $t(17)=4.46, p<.01$, and follow-up, $t(17)=4.61, p<.01$. AF participants reported a significant decrease from baseline to post-training, $t(17)=2.99, p<.01$, but no significant differences from baseline to follow-up, $t(17)=1.79, p>.09$. Data appear in Table 3.

For the FNE, the MANOVA revealed a main effect of Time, $F(2,51)=22.30, p$ $<.01, \eta_{\mathrm{p}}{ }^{2}=.30$, qualified by a significant Time $\mathrm{X}$ Condition interaction, $F(2,51)=7.73$, $p<.01, \eta_{\mathrm{p}}{ }^{2}=.24$. AT participants showed no significant differences from baseline to both post-training, $t(17)=.52, p>.61$, and follow-up, $t(17)=.82, p>.42$. For AnT participants, there were significant decreases from baseline to both post-training, $t(17)=$ $6.01, p<.01$, and follow-up, $t(17)=6.51, p<.01$. For AF participants, there were also significant decreases from baseline to both post-training, $t(17)=3.09, p<.01$, and follow-up, $t(17)=2.95, p<.01$. However, AnT participants had significantly lower scores than AF and AT participants at follow-up. Data appear in Table 3. Emotional responses to a speech task 
Subjective response. The MANOVA revealed a main effect of Time, $F(2,51)=$ $79.72, p<.0001, \eta_{\mathrm{p}}^{2}=.61$, qualified by a significant Time $\mathrm{X}$ Condition interaction, $F(2$, $51)=12.99, p<.0001, \eta_{\mathrm{p}}{ }^{2}=.34$. AT participants showed no significant differences from baseline $(M=74.33, S D=15.19)$ to post-training $(M=68.50, S D=10.53), t(17)=1.40$, $p>.18$. For AnT participants, there were a significant decrease from baseline $(M=75.83$, $S D=13.75)$ to post-training $(M=43.61, S D=19.42), t(17)=6.48, p<.01$. For AF participants, again, there was a significant decrease from baseline $(M=78.28, S D=$ 16.59) to post-training $(M=36.56, S D=21.67), t(17)=6.79, p<.01$.

Psychophysiological responses. For each participant, SCR amplitudes were scored by subtracting the average level during the 15 -sec period prior the speech's information onset from the highest point during the 15-sec period after speech's information onset (Kozak, Foa, \& Steketee, 1988; Dawson, Schell, \& Filion, 1990). Due to a leptokurtotic distribution of SCR amplitude, we used a logarithmic transformation to analysis (Dawson, Schell, \& Filion, 1990). The MANOVA revealed only a significant Time $\mathrm{X}$ Condition interaction, $F(2,51)=10.72, p<.01, \eta_{\mathrm{p}}{ }^{2}=.30$. Although the groups did not differ in their scores at baseline, a one-way ANOVA computed on the score at post-training revealed a significant difference among groups, $F(2,51)=6.20, p<.01$. Tukey HSD post-hoc tests revealed that AnT participants showed significantly smaller reactivity than both AT and AF participants, but no significant differences between AF and AT participants. To examine within-subject effects, we ran follow-up paired-samples $t$ tests on each group separately. For AT as well as for AF participants, there were no significant changes from baseline $\left(M_{A T}=.11, S D_{A T}=.05 ; M_{A n T}=.07, S D_{A n T}=.13\right)$ to post-training $\left(M_{A T}=.10, S D_{A T}=.13 ; M_{A n T}=.13, S D_{A n T}=.08\right)$. In contrast, AnT participants 
showed a significant decrease from baseline $(M=.12, S D=.10)$ to post-training $(M=$ $.20, S D=.18), t(17)=4.165, p<.01$.

Behavioral change. A MANOVA computed on the BASA scores revealed a significant effect of Time, $F(2,51)=5.52, p<.02, \eta_{\mathrm{p}}{ }^{2}=.10$, qualified by a significant Time $\mathrm{X}$ Condition interaction, $F(2,51)=6.41, p<.01, \eta_{\mathrm{p}}{ }^{2}=.20$. Although the groups did not differ in their scores at baseline, a significant difference emerged among groups at post-training, $F(2,51)=5.55, p<.01$. Tukey HSD post-hoc tests revealed that AnT participants displayed significantly less overt signs of anxiety than did AF and AT participants. There were no significant differences between AF and AT participants at post-training.

AT participants exhibited a marginally significant increase in anxiety on the behavioral measure from baseline $(M=81.13, S D=18.78)$ to post-training $(M=87.86$, $S D=14.33), t(17)=2.05, p>.05$. AnT participants showed a significant decrease from baseline $(M=77.19, S D=17.10)$ to post-training $(M=65.69, S D=17.18), t(17)=2.47$, $p<.03$. AF participants showed no significant decrease from baseline $(M=87.77, S D=$ 11.36) to post-training $(M=75.06, S D=23.13), t(17)=1.71, p>.11$.

\section{Mediational Analyses}

To examine whether changes in attentional bias mediated changes in self-report, behavioural, and physiological reactivity to the social stressor, we performed mediational analyses according to MacKinnon, Fairchild, and Fritz's (2007) procedure.

This procedure tests the product of the coefficients for the effects of (a) the independent variable (contrast coded: Attention to positive condition $=+2$, Attention to threat condition $=-3$, Attention flexibility condition $=1$ ) to the mediator (change in 
attention bias from pre-treatment to post-treatment) (alpha), and (b) the mediator to dependent variable when the independent variable is taken into account (beta). This procedure is a variation on the Sobel (1982) test that accounts for the nonnormal distribution of the alpha-beta path through the construction of asymmetric confidence intervals (MacKinnon et al., 2007).

We first examined whether change in attentional bias mediated the impact of treatment condition on the dependent variables (change in scores from baseline to posttreatment for SCR, LSAS, FNE, BASA, and SUDS). Consistent with a statistically significant mediation, the $99 \%$ confidence interval of the indirect path (alpha-beta) did not contain zero, for skin conductance reactivity $(\mathrm{SCR})($ lower limit $=-.005$, upper limit $=$ -.008). Results from the Sobel test supported this conclusion. The same indirect paths for all other measures (LSAS, FNE, BASA, SUDS) overlapped with zero, indicating absence of a significant mediation. Because all variables were measured at the same two points in time (pre-treatment and post-treatment), the direction of causality cannot be definitely determined, and alternative mediational models are possible. Hence, we also examined whether change in SCR to the speech stressor mediated the impact of treatment condition on change in attention bias. However, the $99 \%$ confidence interval of this indirect path overlapped with zero, indicating no significant mediation (lower limit $=-5.20$, upper limit $=1.78$ ). These findings are consistent with the hypothesis that attention training indirectly reduces physiological reactivity to a social stressor via a decrease in attention bias for threatening stimuli.

\section{Discussion}


The primary purpose of this study was to answer two major questions. First, does attention training reduce physiological, self-report, and behavioral measures of anxiety in people with social phobia? Indeed, no previous attention training study had included all three types of measure. Second, does training attentional flexibility produce therapeutic benefits as least as great as those produced by standard attention training which presumably increases the capacity to disengage one's attention from moderately threatening stimuli?

Consistent with the disengagement hypothesis, participants trained to attend to non-threatening stimuli reported lower self-reported social anxiety at post-training and at the 2-week follow-up. Similarly, during the speech task, they manifested lower levels of distress on self-report, physiological, and behavioural indices of distress relative to participants in the other groups. Flexibility training reduced only self-reported distress at posttraining, and even this benefit dissipated by the two-week follow-up.

These results are consistent with studies showing that attention training reduces emotional vulnerability to subsequent stressors (e.g., Amir et al., 2008; See et al., 2009), extending this finding to physiological reactivity. Furthermore, the present study is the first replication of Amir et al.'s (2008) finding of beneficial effects of attention training on behavioral performance during a speech task. Moreover, for participants trained to attend to non-threatening stimuli, reduction in attentional bias toward threat at posttraining mediated change in physiological reactivity to the social stressor.

However, the minimal beneficial effects of flexibility training may be due to its involving the development of two opposing biases that counteract one another. It is 
incumbent on researchers to develop other methods for conceptualizing "flexibility" training that lack this limitation.

Further, regarding cognitive models of Social Phobia, the present data support the idea that selective attention to threatening social stimuli is causally involved in the maintenance of social anxiety (e.g., Amir et al., 2008; Amir et al., 2009; Schmidt et al., 2009). Indeed, these findings bolster the argument that, rather than being a by-product of social phobia, selective attention bias has a causal role in the maintenance of this disorder (Clark, 2001; Clark \& Wells, 1995; Rapee \& Heimberg, 1997).

At a clinical level, these data are consistent with recent developments in cognitive bias modification (e.g., MacLeod et al., 2009) demonstrating that the attention bias for threatening stimuli can be changed and that this change is related to short-term as well as long-term emotional benefits. More precisely, the current double-blind randomized clinical trial adds to a small but growing empirical literature revealing the efficacy of computerized attention training procedure in reducing clinical symptoms in individuals who suffers from social phobia (Amir et al., 2009 Amir et al.,2010, Schmidt et al., 2009). Although the extent of training is modest, totaling no more than a couple of hours over four days, and therapist contact is minimal, clinical benefits occurred on measures of subjective, behavioral, and physiological of anxiety. Further, the 2-week follow-up assessment revealed the maintenance of self-reported benefits.

The present study has limitations. First, our sample size was small, thereby limiting the statistical power of our mediational analysis. Second, we used only skin conductance as the index of change in physiological reactivity. Future research should incorporate other measures, such as heart rate and cortisol release. Third, we used a probe 
discrimination task as a measure of attention bias. However, this task is less than ideal for distinguishing among subcomponents of attentional biases (i.e., difficulties to disengage from threatening stimuli vs. facilitated attention orientation for threatening stimuli). To address this issue, researchers could administer a modified version of the Posner paradigm as an independent measure of attention bias before and after training. In conclusion, the present findings show that training social phobics to attend to non-threatening stimuli reduces self-report, behavioral, and physiological measures of the disorder. Further, the study indicates that attentional biases for threat play a causal role in the maintenance of social phobia. 


\section{References}

American Psychiatric Association. (1994). Diagnostic and statistical manual of mental disorders (4th ed.). Washington, DC: Author.

Amir, N., Beard, C., Taylor, C. T., Klumpp, H., Elias, J., Burns, M., \& Chen, X. (2009). Attention training in individuals with generalized social phobia: A randomized controlled trail. Journal of Consulting and Clinical Psychology, 77, 961-973.

Amir, N., Elias, J., Klumpp, H., \& Przeworski, A. (2003). Attentional bias to threat in social phobia: facilitated processing of threat or difficulty disengaging attention from threat? Behaviour Research and Therapy, 41, 1325-1335.

Amir, N., Weber, G., Beard, C., Bomyea, J., \& Taylor, C. T. (2008). The effects of a single-session attention modification program on response to a public-speaking challenge in socially anxious individuals. Journal of Abnormal Psychology, 117, 860-868.

Beck, A. T., Steer, R. A., \& Brown, G. K. (1996). Beck Depression Inventory manual ( $2^{\text {nd }}$ ed.). San Antonio, TX: Psychological Corporation. French adaptation, 1998, Paris, France: Editions du Centre de Psychologie Appliquée.

Bruchon-Schweitzer, M. \& Paulhan, I. (1993). Adaptation francophone de l'inventaire d'anxiété Trait-Etat (Forme Y) de Spielberger. Paris, France: Editions du Centre Psychologie Appliquée.

Clark, D.M. (1999). Anxiety disorders: why do they persist and how to treat them. Behaviour Research and Therapy, 37, 5-27.

Clark, D. M. \& Wells, A. (1995). A cognitive model of social phobia. In R. G. Heimnberg, M. R. Liebowitz, D. A. Hope, \& F. R., Schneier (Eds.), Social 
Phobia: Diagnosis, assessment, and treatment (pp. 69-93). New York: Guilford Press.

Dawson, M. E., Schell, A. M., \& Fillion, D. L. (1990). The electrodermal system. In J. C. Cacioppo \& L. G. Tassinary (Eds.). Principles of psychophysiology: Physical, social, and inferential elements, (pp. 295-324). New York: New York University Press.

Douilliez, C., Baeyens, C., \& Philippot, P. (2008). French validation of the Fear of Negative Evaluation Scale and the Social Avoidance and Distress Scale. Revue francophone de Clinique Comportementale et Cognitive, 13, 1-12.

Fox, E., Russo, R., Bowles, R., \& Dutton, K. (2001). Do threatening stimuli draw or hold visual attention in subclinical anxiety? Journal of Experimental Psychology: General, 130, 681-700.

Kozak, M. J., Foa, E. B., \& Steketee, G. (1988). Process and outcome of exposure treatment with obsessive-compulsives - Psychophysiological indicators of emotional processing. Behavior Therapy, 19, 157-169.

Grey, S. J., \& Smith, B. L. (1984). A comparison between commercially available electrode gels and purpose-made gel, in the measurement of electrodermal activity. Psychophysiology, 21, 551-557.

Heimberg, R. G., et al. (1999). Psychometric properties of the Liebowitz Social Anxiety Scale. Psychological Medicine, 29, 199-212.

Lecrubier, Y., Weiller, E., Bonora, L. I., Amorin, P., \& Lépine, J.-P. (1998). French adaptation of the Mini International Neuropsychiatric Interview (MINI 5.0.0.). Internal report, Unité INSERM 302. Hôpital de la Salpétrière, Paris, France. 
Li, S. W., Tan, J. Q., Qian, M. Y., \& Liu, X. H. (2008). Continual training of attentional bias in social anxiety. Behaviour Research \& Therapy, 46, 905-912.

Liebowitz, M. R. (1987). Social phobia. Modern Problems of Pharmacopsychiatry, 22, $141-173$.

Lundh L.G., \& Öst L.G. (2001). Attentional bias, self-consciousness and perfectionism in social phobia before and after cognitive-behaviour therapy. Scandinavian Journal of Behaviour Therapy, 30, 4-16.

MacKinnon, D. P., Fairchild, A. J., \& Fritz, M. S. (2007). Mediation analysis. Annual Review of Psychology, 58, 593-614.

MacKinnon, D. P., Lockwood, C. M., Hoffman, J. M., West, S. G., \& Sheets, V. (2002). A comparison of methods to test mediation and other intervening variable effects. Psychological Methods, 7, 83-104.

MacLeod, C., Koster, E. H. W., \& Fox, E. (2009). Whither cognitive bias modification research? Commentary on the special section articles. Journal of Abnormal Psychology, 118, 89-99.

Macleod, C., Mathews, A., \& Tata, P. (1986). Attentional bias in emotional disorders. Journal of Abnormal Psychology, 95, 15-20.

MacLeod, C., Rutherford, E., Campbell, L., Ebsworthy, G., \& Lin, H. (2002). Selective attention and emotional vulnerability: Assessing the causal basis of their association through the experimental manipulation of attentional bias. Journal of Abnormal Psychology, 111, 107-123.

Mathews, A. \& MacLeod, C. (1994). Cognitive approaches to emotion and emotional disorders. Annual Review of Psychology, 45, 25-50. 
Mathews, A. \& MacLeod, C. (2002). Induced processing biases have causal effects on anxiety. Cognition and emotion, 16, 331-354.

Mogg, K., Bradley, B. P., Millar, N., \& White, J. (1995). A follow-up study of cognitive bias in generalized anxiety disorder. Behaviour Research Therapy, 33, 927-935.

Mogg, K., Holmes, A., Garner, M., \& Bradley, B. P. (2008). Effects of threat cues on attentional shifting, disengagement and response slowing in anxious individuals. Behaviour Research and Therapy, 46, 656-667.

Mogg, K., Philippot, P., \& Bradley, B. P. (2004). Selective attention to angry faces in clinical social phobia. Journal of Abnormal Psychology, 113, 160-165.

Mulac, A. \& Sherman, R. (1977). Behavioral assessment of speech anxiety. Quarterly Journal of Speech, 60, 134-143.

Musa, C. N., Kostogianni, \& Lépine, J. P. (2004). The fear of negative evaluation scale (FNE): Psychometric properties of the French version. Encephale-Revue De Psychiatrie Clinique Biologique Et Therapeutique, 30, 517-524.

Pishyar, R., Harris, L. M., \& Menzies, R. G. (2004). Attentional bias for words and faces in social anxiety. Anxiety Stress and Coping, 17, 23-36.

Posner, M. I. (1980). Orienting of attention. Quarterly Journal of Experimental Psychology, 32, 3-25.

Rapee, R. M. \& Heimberg, R. G. (1997). A cognitive-behavioral model of anxiety in social phobia. Behaviour Research and Therapy, 35, 741-756.

Reese, H. E., McNally, R. J., Najmi, S., \& Amir, N. (2010). Attention training for reducing spider fear in spider-fearful individuals. Journal of Anxiety Disorders, 24, 657-662. 
Schmidt, N. B., Richey, J. A., Buckner, J. D., \& Timpano, K. R. (2009). Attention training for generalized social anxiety disorder. Journal of Abnormal Psychology, 118, 5-14.

See, J., MacLeod, C., \& Bridle, R. (2009). The reduction of anxiety vulnerability through the modification of attentional bias: A real-world study using a home-based cognitive bias modification procedure. Journal of Abnormal Psychology, 118, $65-75$

Sobel, M. E. (1982). Asymptotic intervals for indirect effects in structural equations models. In S. Leinhart (Ed.), Sociological methodology (pp. 290-312). San Francisco, Jossey-Bass.

Somerville, L.H. et al. (2004). Human amygdala responses during presentation of happy and neutral faces: correlations with state anxiety. Biological Psychiatry, 55, 897-903.

Spielberger, D. C., Gorsuch, R. L., Lushene, R., Vagg, P. R., \& Jacobs, G. A. (1983). Manual for the State-Trait Anxiety Inventory. Palo Alto, CA: Consulting Psychology Press.

Watson, D., \& Friend, R. (1969). Measurement of social-evaluative anxiety. Journal of Consulting and Clinical Psychology, 33, 448-457.

Wells, A. (2000). Emotional disorders and metacognition: innovative cognitive therapy. Chichester, UK: Wiley.

Wolpe, J. (1958). Psychotherapy by reciprocal inhibition. Stanford, CA: Stanford University Press. 
Yao, S. N., Note, I., Fanget, F., Albuisson, E., Bouvard, M., Jalenques, I., \& Cottraux, J. (1999). Social anxiety in social phobics: Validation of Liebowitz's social anxiety scale - French version. Encephale-Revue De Psychiatrie Clinique Biologique Et Therapeutique, 25(5), 429-435.

Yiend, J. (2010). The effects of emotion on attention: A review of attentional processing of emotional information. Cognition and Emotion, 24, 3-47.

Yoon, K. L., \& Zinbarg, R. E. (2008). Interpreting neutral faces as threatening is a default mode for socially anxious individuals. Journal of Abnormal Psychology, 117, 680-685. 
Table 1

Participants Characteristics as a Function of Group Allocation (Standard Deviations in Parentheses)

\begin{tabular}{lccc}
\hline & Attend to Threat & Attend to Non-threat & Attend flexibly \\
\cline { 2 - 4 } Age & $22.17(3.93)$ & $22.11(3.41)$ & $21.44(2.23)$ \\
\% female & 55.6 & 50.0 & 44.4 \\
Years of Education & $15.94(1.39)$ & $15.78(1.06)$ & $16.22(1.00)$ \\
BDI-II & $12.44(4.55)$ & $9.61(3.45)$ & $9.50(5.27)$ \\
STAI-T & $33.22(10.30)$ & $28.17(7.43)$ & $34.00(7.17)$ \\
FNE & $22.72(3.51)$ & $22.50(2.77)$ & $23.50(4.50)$ \\
LSAS & $80.50(5.75)$ & $81.50(4.46)$ & $79.39(4.38)$
\end{tabular}

Note. BDI-II is Beck Depression Inventory-II, STAI-T is Spielberger State-Trait Anxiety Inventory-Trait, FNE is Fear of Negative Evaluation scale; LSAS is Liebowitz Social Anxiety Scale. 
Table 2

Bias Score (d) as a Function of Group Allocation (Standard Deviations in Parentheses) and Times

\begin{tabular}{lccc} 
& Attend to Threat & Attend to Non-threat & Attend flexibly \\
\cline { 2 - 4 } Baseline & $30,49(59.13)$ & $28,69(28.50)$ & $23,34(51.74)$ \\
Post-training & $33,23(23.99)$ & $-23,32(13.71)^{* * *}$ & $38,79(29.71)$
\end{tabular}

Note. Attend to Threat $=$ an attentional bias away from threatening material; attend to non-threat $=$ an attentional bias toward threatening material; attend flexibility $=$ attention flexibility condition. “*” indicates a significant difference between pre- and postintervention in that group according to paired $t$-test comparisons. For all types of measures, there were no significant differences in the first session between groups according $t$-test comparisons. $* * * p<.001$. 
Table 3

Changes in Self-reported measures of Social Anxiety as a Function of Condition and Time (Standard Deviations in Parentheses)

\begin{tabular}{|c|c|c|c|c|c|c|c|c|c|}
\hline & \multicolumn{3}{|c|}{ Attend to Threat } & \multicolumn{3}{|c|}{ Attend to Non-threat } & \multicolumn{3}{|c|}{ Attend flexibly } \\
\hline & Baseline & $\begin{array}{l}\text { Post- } \\
\text { training }\end{array}$ & Follow-up & Baseline & $\begin{array}{l}\text { Post- } \\
\text { training }\end{array}$ & Follow-up & Baseline & $\begin{array}{l}\text { Post- } \\
\text { training }\end{array}$ & Follow-up \\
\hline LSAS & $\begin{array}{c}80.50 \\
(24.39)\end{array}$ & $\begin{array}{c}77.06 \\
(25.76)\end{array}$ & $\begin{array}{c}74.28 \\
(21.77)\end{array}$ & $\begin{array}{c}81.50 \\
(18.92)\end{array}$ & $\begin{array}{c}64.61 \\
(22.33)^{* * *}\end{array}$ & $\begin{array}{c}51.06 \\
(25.40) * * *\end{array}$ & $\begin{array}{c}79.39 \\
(18.60)\end{array}$ & $\begin{array}{c}62.89 \\
(22.80)^{* *}\end{array}$ & $\begin{array}{c}71.22 \\
(15.88)\end{array}$ \\
\hline FNE & $\begin{array}{l}22.72 \\
(3.51)\end{array}$ & $\begin{array}{l}22.33 \\
(4.68)\end{array}$ & $\begin{array}{l}22.11 \\
(3.16)\end{array}$ & $\begin{array}{l}22.50 \\
(2.79)\end{array}$ & $\begin{array}{c}19.06 \\
(3.84)^{* * *}\end{array}$ & $\begin{array}{c}14.39 \\
(5.38)^{* * *}\end{array}$ & $\begin{array}{l}23.50 \\
(4.50)\end{array}$ & $\begin{array}{c}19.67 \\
(5.18)^{* *}\end{array}$ & $\begin{array}{c}20.17 \\
(3.28)^{* *}\end{array}$ \\
\hline
\end{tabular}

Note. Attend to Threat = an attentional bias away from threatening material; attend to non-threat $=$ an attentional bias toward threatening material; attend flexibility = attention flexibility condition; LSAS = Liebowitz Social Anxiety Scale; FNE = Fear of Negative Evaluation. “*” indicates a significant difference between pre- and post- intervention in that group according to paired $t$-test comparisons. For all types of measures, there were no significant differences in the first session between groups according $t$-test comparisons $* * p<.01 . * * * p<.001$. 